\title{
A progressive postresection walking program significantly improves fatigue and health-related quality of life in pancreas and periampullary cancer patients.
}

\author{
Theresa Yeo \\ Thomas Jefferson University School of Nursing \\ Sherry A Burrell \\ Rutgers, The State University of New Jersey \\ Patricia K Sauter \\ Thomas Jefferson University Hospital \\ Eugene P Kennedy

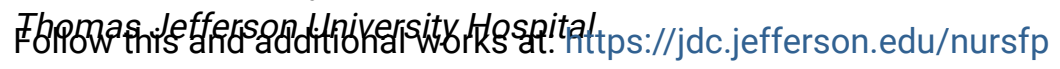 \\ Irisastlofitle Nursing Commons, and the Surgery Commons

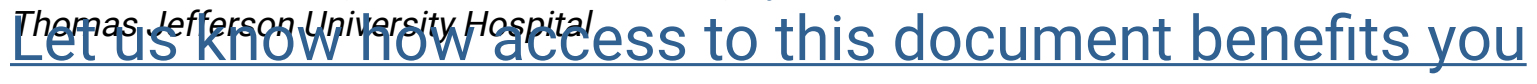

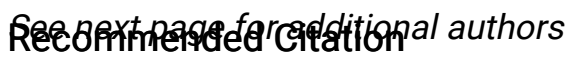

Yeo, Theresa; Burrell, Sherry A; Sauter, Patricia K; Kennedy, Eugene P; Lavu, Harish; Leiby, Benjamin E; and Yeo, Charles, "A progressive postresection walking program significantly improves fatigue and health-related quality of life in pancreas and periampullary cancer patients." (2012). College of Nursing Faculty Papers \& Presentations. Paper 50.

https://jdc.jefferson.edu/nursfp/50

This Article is brought to you for free and open access by the Jefferson Digital Commons. The Jefferson Digital Commons is a service of Thomas Jefferson University's Center for Teaching and Learning (CTL). The Commons is a showcase for Jefferson books and journals, peer-reviewed scholarly publications, unique historical collections from the University archives, and teaching tools. The Jefferson Digital Commons allows researchers and interested readers anywhere in the world to learn about and keep up to date with Jefferson scholarship. This article has been accepted for inclusion in College of Nursing Faculty Papers \& Presentations by an authorized administrator of the Jefferson Digital Commons. For more information, please contact: JeffersonDigitalCommons@jefferson.edu. 


\section{Authors}

Theresa Yeo, Sherry A Burrell, Patricia K Sauter, Eugene P Kennedy, Harish Lavu, Benjamin E Leiby, and Charles Yeo 


\title{
As submitted to \\ Journal of the American College of Surgeons
}

And later published as:

\begin{abstract}
A progressive post-resection walking program significantly improves fatigue and health-related quality of life in pancreas and periampullary cancer patients
\end{abstract}

\author{
April 2012, Volume 214 Issue 4, pp. 463-75
}

\section{DOI: 10.1016/j.jamcollsurg.2011.12.017}

\author{
Theresa P. Yeo, Ph.D., MPH, MSN, AOCNP ${ }^{1,2,3}$ \\ Sherry A. Burrell, RN, CNE, ACNS-BC ${ }^{5}$ \\ Patricia K. Sauter, MSN, ACNP ${ }^{2,3}$ \\ Eugene P. Kennedy, MD, FACS ${ }^{2,3}$ \\ Harish Lavu, MD, FACS ${ }^{2,3}$ \\ Benjamin E. Leiby, $\mathrm{PhD}^{4}$ \\ and \\ Charles J. Yeo, MD, FACS ${ }^{2,3}$
}

From the Jefferson School of Nursing ${ }^{1}$, Jefferson Department of Surgery ${ }^{2}$, Jefferson Pancreas,

Biliary and Related Cancer Center ${ }^{3}$, Jefferson Division of Biostatistics, Department of

Pharmacology and Experimental Therapeutics ${ }^{4}$ and Rutgers, The State University of New Jersey, School of Nursing 5

Thomas Jefferson University

Philadelphia, PA 19107

Presented at the Southern Surgical Association, $123^{\text {rd }}$ Annual Meeting,

Hot Springs, Virginia, December 2011 and

previously partially presented as a Poster of Note at the Pancreas Club, Chicago, IL, May 2011

Running Title: Walking and Fatigue in Pancreas Cancer Patients

Key Words: pancreas and periampullary cancer, pancreatectomy, health related quality of life, fatigue, walking, exercise

Funded by a Jefferson School of Nursing Faculty Seed Money Research Award 
Corresponding Author:

Theresa P. Yeo, PhD, MPH, MSN, AOCNP

Associate Professor

Program Director: Advanced Practice Oncology Nursing

Jefferson School of Nursing

Department of Surgery

Thomas Jefferson University

Philadelphia, PA 19107

theresa.yeo@jefferson.edu

\section{ABSTRACT}

Introduction: As patients with pancreas and periampullary cancer (PPC) experience improved survival rates and longevity, the focus shifts towards living life while surviving cancer. Fatigue is the most commonly reported symptom in all cancer patients. Exercise has been found to effectively decrease fatigue levels and improve physical functioning in cancer patients.

Methods: One hundred and two patients with resected PPC consented to participate in this study and were randomized to either an Intervention Group (IG) or a Usual Care Group (UCG). Subjects completed visual analog scales, the FACIT-Fatigue Scale (FFS) and the Short Form 36v2® after surgery and again 3 to 6 months after hospital discharge.

Results: Patients in the IG and UCG were comparable with regard to demographics, comorbidities, cancer type and staging, type of resection, pre-op fatigue and pain levels, adjuvant therapy and baseline walking distance. Patients in the IG had significantly improved scores on the FFS at study completion, improved fatigue and pain scores, as well as overall physical functioning and mental health composite scores. At study completion, participants in the IG were walking twice as far and were significantly more likely to have continued walking or another form of exercise as compared to the UCG. Using hierarchical cluster analysis three mutually exclusive symptom groupings were identified in the cohort. Kaplan-Meier survival analysis did not indicate an overall survival benefit for the IG.

Conclusion: This is the first prospective, randomized and controlled trial to report that participation in a home walking program confers a significant benefit in resected PPC patients 
with regard to fatigue levels, physical functioning and HRQOL. (ClinicalTrials.gov number, NCT00902759). 


\section{INTRODUCTION}

Approximately 50,000 new cases of pancreas and peri-ampullary (PPC) adenocarcinoma occur annually in the United States (1). Surgical resection offers the possibility of long-term survival with 5-year survival rates varying from 10 to 50\%, depending upon such factors as pathologic staging, patient performance status and individual institutional volumes (2 - 8). As patients with PPC experience improved survival rates and longevity, the focus shifts towards living life while surviving cancer, managing adverse symptoms, and improving physical functioning and quality of life (QOL).

Fatigue and pain are the most commonly reported symptoms by all cancer patients including those undergoing chemotherapy (CT) and radiation therapy (RT). Fatigue is the most universally reported symptom and is considered by many patients as more distressing than pain $(9,10)$. For example, when 600 postings from pancreas cancer patients and their families in a monitored Internet pancreas cancer Frequently Asked Questions chat room were analyzedpain, fatigue, and anorexia were the most frequently reported symptoms by post-operative patients as well as those who did not have surgery (11). Other investigations have reported fatigue, pain, weight loss, nausea, anorexia, constipation, diarrhea, insomnia, depression, and anxiety as the most prevalent symptoms reported by PPC patients at all stages of disease $(6,12$ $15)$.

Fatigue is defined in this study as cancer- related fatigue (CRF), described by the National Comprehensive Cancer Network (NCCN) as "a distressing persistent, subjective sense of physical, emotional, and/or cognitive tiredness or exhaustion related to cancer or cancer treatment that is not proportional to recent activity and interferes with usual functioning", and is also referred to as rest-resistant fatigue $(16,17)$. CRF typically leads to decreased activity levels, contributes to a reduction in one's overall QOL and is often accompanied by anxiety and depression. CRF is known to be a complex process that is influenced by muscle catabolism and 
protein loss, weight loss, a decline in neuromuscular efficiency, de-conditioning, cancer treatment factors, decreased sleep, insomnia and anemia $(17,18)$.

Exercise, defined as planned, structured and repetitive body movement, has been found to effectively decrease fatigue levels and improve functional capacity in patients with breast and prostate cancer $(19,20)$. Exercise increases muscle mass, promotes flexibility and elevates mood, all of which improve one's ability to cope following cancer surgery and during adjuvant therapy. Cancer patients who exercise on a regular basis may reduce their fatigue level by one-half. Although it may seem paradoxical, fatigue that results from exercise helps reduce CRF by improving the quality and perhaps the quantity of restful sleep.

A 2011 systematic review by Wanchai et al reviewed 28 of the 89 published studies on the effect of non-pharmacologic supportive strategies on CRF in breast cancer patients (21). The results showed that home-based exercise, supervised exercise, education, sleep therapy, and complementary therapy were effective strategies to improve QOL and reduce CRF among breast cancer survivors. The majority of the published studies on fatigue and quality of life have been conducted in patients with breast cancer, followed by stem cell transplant recipients and lastly prostate cancer survivors. By contrast, this is the first report of a randomized controlled trial that prospectively evaluated the effects of a home-based walking program in post-resection PPC patients on the primary outcomes of CRF, physical function and QOL.

A secondary aim of this trial was to explore the occurrence of persistent self-reported symptoms in individuals with PPC following surgical resection. There is a growing body of evidence that individuals affected by cancer often do not present with one isolated symptom, but rather with multiple, concurrent symptoms or a symptom cluster (the presence of two or more symptoms occurring simultaneously, which may or may not share the same etiology) (22-24). Several studies have examined the occurrence of symptoms in patients following resection of the pancreas and have found that the most common issues are: foul bowel movements, thirst, 
frequent urination, poor blood glucose control, heartburn, nausea and vomiting, weight loss, abdominal pain, fatigue, and muscle weakness $(6,15,25-27)$. This study was conceptualized using the Levine Conservation of Energy Theory as the theoretical background (28). In this model, fatigue represents ongoing adaptation to the illness state and can be a measurable outcome. 


\section{METHODS}

Trial design: This investigator-initiated study used a two group, prospective, randomized controlled design which consisted of a walking intervention group and a comparison usual care control group. The study was approved by the Clinical Cancer Research Review Committee of the Jefferson Kimmel Cancer Center and by the Thomas Jefferson University Institutional Review Board prior to enrollment of patients and is registered with ClinicalTrials.gov (NCT00902759).

A convenience sample of men and women $(n=102)$ ages 18 years or older with resected PPC at the Thomas Jefferson University Hospital Department of Surgery consented to participate in this randomized controlled trial. Eligible patients, post-resection of PPC (adenocarcinoma only) via pancreatectomy, were referred to the principal investigator (TPY) by three pancreatobiliary surgeons (EPK, HL, CJY). Patients were not eligible if Stage IV metastatic disease was found at the time of surgery, if the final pathology revealed other than adenocarcinoma, or if co-morbidities such as severe arthritis, dizziness or inability to walk prevented the patient from actively participating in a walking program. Identified patients meeting the eligibility criteria were approached while hospitalized following resection or at their first post-operative office visit. After obtaining informed consent, subjects were randomized without stratification using a computer-generated randomization schedule and the sealed envelope technique to either an Intervention Group (IG) or a Usual Care Group (UCG) in a 1:1 ratio (See Figure 1). All subjects had either resection alone or resection plus adjuvant CT and/or RT between April 2009 and January 2011. The study was closed to active follow-up on May 26, 2011, however the survival data were updated through September 6, 2011.

Outcome Measures: The primary study endpoints were to determine the effects of a walking program on cancer-related fatigue, physical functioning and QOL after three to six months of study participation using reliable and validated measurement tools. Secondary endpoints were 
overall survival and an exploration of the symptom burden and clustering in this patient population.

Sample Size Calculation: A sample size of 100 (50 in each group) was deemed necessary based on the assumption of approximately a $40 \%$ improvement in fatigue, physical functioning, and QOL in the UCG versus an anticipated 70\% improvement in the IG, based on previous studies (19). Using the sample size table by Simon (29) for a one-tailed test, small to moderate effect size due to the exercise intervention, power $(1-\beta)$ of 0.80 and a level of significance $(\alpha)$ of 0.05 , it was determined that a minimum of 80 subjects would be needed to detect a difference if one existed between the groups. With 80 patients as a minimum target, additional patients were recruited, anticipating some degree of study dropout, problems with the exercise program and some patient loss to follow-up.

Methodology: Following an initial meeting in which the study protocol and procedures were reviewed with all investigators, regular meetings were held with the primary data collectors. A script was developed for the monthly follow-up telephone calls in order to standardize the questions asked. Two investigators (TPY and SAB) conducted the majority of the follow-up phone calls.

Study screening procedures included a baseline assessment on all subjects either in the hospital or in the office, consisting of a targeted physical examination and an observed walk using a pre-determined and pre-measured route on the hospital floor or in the outpatient clinic. The PI or a co-investigator accompanied the patient during the baseline walk and recorded the distance walked (in feet) and/or the amount of time walked. Emergency equipment and a chair were available should they be needed during the walk. Fatigue and Pain Visual Analog Scales (FVAS and PVAS), the FACIT-Fatigue Scale (FFS) questionnaire and the Medical Outcomes Study Short Form-36 (SF-36v2@) questionnaire were completed by patients at study entry, but 
prior to randomization. The same assessment tools were completed a minimum of 3 months after hospital discharge or at the completion of adjuvant therapy (See Figure 2).

Subjects randomized to the IG were given a graduated walking prescription for a home walking program based on Every Step Counts-A Walking Exercise Program for Persons Living with Cancer, published by Mock in 1997 (30). Permission to reproduce this booklet and to use the program was granted by the Johns Hopkins University School of Nursing (personal communiqué, 2007). The graduated walking prescription includes three phases and was individualized for each patient based on their baseline walk and general physical condition (See Figure 3). Participants were instructed to walk at home, in their neighborhood (if safe), at an exercise gym or in a shopping mall for as far as possible on a level surface, wearing appropriate footwear. They were instructed to stop or slow down if they experienced physical symptoms such as uncomfortable shortness of breath, chest pain, or bodily pain. The goal was to eventually walk for 90 - 150 minutes per week in three to five sessions. In order to reduce the perceived burden of the program, participants were not asked to routinely take their pulse or to record their perceived exertion, but the exercise booklet describes how to do this if they chose to.

Patients in the IG were also asked to keep a weekly diary-type log of the number of minutes or the approximate distance walked at each session and to record in the log any adverse symptoms experienced during the week. Self-addressed, stamped envelopes were provided and additional $\log$ sheets were mailed to the participants as needed. Patients in the IG also received a monthly follow-up telephone call from either the PI or a co-investigator for two purposes: to provide a reminder to mail in the log sheets and to assess their current status using a predetermined set of standardized questions. These standardized questions included their current medical condition, adherence with the walking program, the amount and type of exercise performed, their current FVAS and PVAS rating, chemotherapy or radiation therapy being 
received, and the presence or absence of the following 10 symptoms: fatigue, pain, shortness of breath, weight loss, anorexia, diarrhea, constipation, weakness, insomnia, and depression.

This 10 symptom profile survey was based on a literature review of the most commonly reported symptoms in patients with PPC $(6,11,12-15,24-27)$, and was administered to the IG during monthly follow-up phone calls and upon final follow-up. The UCG received the followup 10 symptom profile survey only upon final follow-up. To ensure the consistency of our follow-up symptom survey findings, subject responses were crossed-checked with FACITfatigue scores, pain and fatigue visual analog scores, and the SF-36 Health Survey responses. During the monthly follow-up calls, if an IG patient was feeling particularly low due to the administration of chemotherapy or radiation therapy that day, we called them back the following week, (recognizing that symptoms are heavily influenced by the effects of treatment). We were also consistent about the time of day that we called and found that the afternoon between 4 - 5 PM was a reliable time to reach patients and a time when they were willing to talk with us. This allowed patients to reflect on their symptoms over the course of the day increasing the likelihood that their responses reflected the average daily symptom ratings.

The patients in the UCG did not receive a monthly phone call. They received a study follow-up telephone call from the PI or co-investigator, beginning 3 months following entry into the study. In some instances it took several months to reach patients in the UCG group for follow-up. At the time of follow-up, they were asked to complete the 10 symptom profile survey, as well as the FVAS, PVAS, FFS, and the SF-36v2@ assessment tools. The FFS and SF$36 \mathrm{v} 2 ®$ questionnaires were completed on the telephone with the investigator or if so requested, the forms were mailed to the subject for completion.

\section{TOOLS UTILIZED:}

Exercise Program: The Every Step Counts graduated walking program was implemented in this study with minor modifications for resected PPC patients (30) (See Figure 3). The 
modifications included: reducing the length of the program from six months to three months, and recommending a more gradual increase in brisk walking time to 10 minutes in the first month, 20 minutes in the second month and 25-30 minutes in the third month. An individual "Exercise Prescription" was given to the IG patients at the time of entry into the study, as well as a copy of the Every Step Counts booklet.

Fatigue and Pain Visual Analog Scales (FVAS and PVAS): Visual numeric rating scales (visual analog scales - VAS) for pain and fatigue were used in the study and are valuable for obtaining ordinal measures of pain and fatigue and to serve as exploratory measurement tools. Numeric scales can be corroborated with targeted tools that address more than just severity of the symptom (31). Fatigue and pain VAS were obtained at baseline and at each follow-up phone call with the IG, and at the end of the study in both the UCG and the IG.

FACIT-Fatigue Scale (FFS): The FFS is a standardized 13-item fatigue subscale of the FACTF developed in 1997 from 32 items submitted by five medical experts and 221 items from 14 cancer patients (32). It has high test-retest reliability $(r=0.87)$ and internal consistency $(\alpha=$ 0.95 and 0.93 ), supporting its ability to be used as an independent, one-dimensional measure of fatigue. One of the advantages of the FFS is that it asks patients to consider their answers in the context of the last 7 days. This approach is considered to be representative of average feelings and is less influenced by the high and low ends of the fatigue spectrum or by treatment effects. The FFS was administered at baseline, at each follow-up phone call with the IG, and at the end of the study in the both UCG and the IG.

ECOG Performance Status Rating Scale: The ECOG performance status rating scale is a six level ( 0 , fully functional -5 , deceased) assessment tool that provides a measure of physical function by assessing the degree to which cancer patients are able to participate in the activities of daily living, as well as their need for rest (33). It is a global index which has been widely used 
in clinical trials to assess the functional capability of cancer patients. We used the ECOG level for a baseline and follow-up measure of performance ability in both the IG and UCG.

Short Form-36v2® Health Survey (SF-36v2®): The SF-36v2® is a comprehensive, valid and reliable multipurpose general health survey $(34,35)$. It is not targeted to a specific disease, age group or treatment group, but rather assesses health concepts that are universally valued (35). It consists of 36 questions addressed through eight domains (cognitive functioning, sleep, health distress, social support, family and marital functioning, sexual functioning, physical symptoms and psychological symptoms) and 1 transitional question. Two psychometrically based composite scores, the Physical Component Summary (PCS) and the Mental Component Summary (MCS) are generated using the QualityMetric Health Outcomes TM scoring Software (Version 3.0.21129. Copyright 2004-2009). These two composite scores are considered a valid representation of health-related quality of life (HRQOL) and indicate the extent to which physical or mental problems interfere with the eight domains of life (36). The SF-36v2® is based on norms developed from the 1998 National Survey of Functional Health Status, and it measures a 4 week recall period. Of the 36 questions on the questionnaire, 14 questions address physical functioning, 2 questions assess bodily pain, 5 questions reflect general health, 4 questions reflect one's vitality, 2 questions address social functioning, 11 questions concern mental health, and one question assesses health transition and estimates the average change in health status in the year prior to administration. However the health transition question is not used in scoring the scales or in the summary measures. The SF-36v2® has been studied in multiple investigations and reliability coefficients range from 0.8 to 0.93 (37). The two composite measures, PCS and MCS, are reliable composite measures of the domains included in their compilation and together can be used to indicate one's HRQOL. The percent of the sample at-risk for depression is also calculated based on the normogram. Lower scores on the PCS reflect limitations in self-care, physical function and social and role activities, frequent tiredness, 
and rating of one's health as poor. Lower scores on the MCS indicate frequent psychological distress and social and role disability due to emotional issues. Obtaining a high PCS or MCS requires more than just freedom from physical limitations and social and role disability; it requires that the respondent rated their health as "excellent". Rating one's overall health as very good, good, fair or poor affects both of these composite measures. Likewise rating one's health as "excellent" is not sufficient for a very high score. The PCS and MCS weight the personal and social implications considerably.

Monthly Exercise Diary: Patients in the IG were asked to use a self-reported diary-log to record the length of time and/or the approximate distance that they walked or exercised each week. They were also asked to record any adverse and bothersome symptoms that they experienced either during walking or at other times. The logs were to be mailed to the PI on a monthly basis.

Statistical Analysis: All data were initially entered into an Excel database and later transferred to Stata-Version 8 and SAS Version 9.2 statistical packages for data analyses. Baseline descriptive statistics were computed for the IG and UCG. $\chi^{2}$ testing and linear and logistic regression models were used for comparing continuous and categorical variables. T-tests and paired t-tests were used to compare the continuous variables. Survival analysis was conducted using the Kaplan-Meier method and the Cox proportional hazards model. Symptom analysis was performed using hierarchical cluster analysis. Distances between subjects were calculated using the Gower dissimilarity measure, and clustering was performed using the Centroid method. Statistical significance was set at $p \leq 0.05$. Data were analyzed according to the intention to treat principle. 


\section{RESULTS}

During the study, 165 patients were potentially eligible for participation, based upon their final pathology and operative procedure performed (Figure 1). Forty seven (47) were not approached due to complications limiting recovery, social issues, administrative lapses or clear inability to exercise. The remaining one hundred eighteen (118) patients were approached to participate in the study. Eight (8) patients declined participation because they felt too ill, were not interested or felt that they were not fluent enough in English to participate. Thus, 110 patients were enrolled in the study; 102 had sufficient data to be included in the analysis. Twenty-three subjects were censored during the study period; 15 died during the study period, 3 withdrew from the study, and 5 were lost to follow-up. At the last survival follow-up (9/6/11), the median follow up was 19 months, and there were 27 confirmed deaths in the total cohort, 12 deaths in the IG and 15 deaths in the UCG. Seventy-nine (79) of the 102 patients enrolled completed the study.

\section{Demographic and Clinical Characteristics (Table 1):}

The allocation yielded two groups with comparable demographic and clinical characteristics, indicating effective randomization. The mean age of patients in the IG was 66 years and 67 years in the UCG. Half of the patients were men, most were white, and over $90 \%$ of the patients had a pathologic diagnosis of primary pancreatic ductal adenocarcinoma. Most patients had either Stage $2 \mathrm{a}$ or $2 \mathrm{~b}$ tumors $(73 \%$ and $72 \%$ in the IG and UCG, respectively) and over $90 \%$ in each group had a pylorus-preserving pancreaticoduodenectomy. Co-morbidities in the groups were similar with hypertension, diabetes, coronary artery disease and a previous history of cancer being the most commonly identified conditions. Over half of each group selfidentified as either current or previous cigarette smokers. There were no significant differences between groups with regard to pre-operative serum values of albumin, glucose, total bilirubin, hemoglobin and the tumor markers CEA and CA 19-9. The only parameter that was 
significantly different was study entry body mass index (BMI). The BMI in the IG was 28 , as compared to 26 in the UCG $(\mathrm{p}=0.02)$. Data were available on adjuvant chemotherapy for 53 IG patients and 46 UCG patients, with 5-flourouracil and gemcitabine being the chemotherapeutic agents received most often. During the study period, there 7 deaths in the IG compared to 8 deaths in the UCG. As concerns follow-up, $74 \%$ of the IG and $71 \%$ of the UCG completed the minimum 3 month follow-up. A number of patients were followed for up to a year or more, so as to obtain as long as possible follow-up.

\section{Walking Program (Table 2):}

On the initial baseline walk, the UCG on average walked further than the IG (1404 feet versus 913 feet, $\mathrm{p}=$ N.S.). The mean distance walked at the time of the first month of follow-up by the IG was 2.5 miles. This distance decreased to 1.1 miles at the month 2 interval, which often corresponded to the timing of administration of adjuvant therapy. The distance walked increased to 2 miles at the final 3 month walk for the IG. At the end of the study period, the IG was walking twice as far as the UCG ( 2 miles versus 1 mile, $p=0.1,3-5$ times per week) and the IG were significantly more likely ( $80 \%$ vs. $58 \%$ ) to still be walking or engaged in another form of exercise $(p=0.04)$.

\section{Fatigue (Table 3):}

Fatigue scores were assessed at baseline and at a mean follow-up of 3 to 6 months using the Fatigue VAS and the FFS. At entry into the study, $85 \%$ of all participants reported moderate to severe fatigue (FVAS greater than 5). Baseline FFS scores (range 0-52) were not significantly different between the groups with a mean score of 27 in the IG and 30 in the UCG. Comparing baseline and end-of-study scores between groups, only patients in the IG had significantly improved fatigue scores by both fatigue measures at the end of the study, as compared to their own baseline $(\mathrm{p}=0.05)$. Higher FFS scores tended to correlate with lower stage of disease. Those reporting lower fatigue scores also had the lowest pain scores. 


\section{Pain (Table 3):}

Patients in both groups reported pain in the mild range (PVAS $=2.9)$ at entry into the study, which significantly improved in both groups (to 1.6 in the IG and to 1.8 in the UCG) by the end of the study (both $p=0.05$ ).

\section{ECOG Stage (Table 3):}

At entry to the study, $95 \%$ of the IG were ECOG levels 1 and 2, while in the UCG, $87 \%$ were ECOG levels 1 and 2, and 6\% were ECOG 0. The baseline ECOG scores for both groups indicate that most participants were somewhat restricted in performing physically strenuous activity but were ambulatory and able to perform some light work. Neither the pre-ECOG scores nor the post-ECOG scores differed significantly between the groups, but there was a trend towards less self-care and less ability to carry out any work activities in the UCG at the end of study, and their ECOG score rose at study end (1.5 to 1.8), while the ECOG score in the IG fell (1.6 to 1.5$)$.

\section{Short Form-36v2® (Tables 4 and 5):}

The overall quality of the SF-36v2® questionnaire data was acceptable for both the IG and UCG, with the data quality indicators for completeness of data, responses in range, consistency, internal consistency, discriminate validity, and reliability ranging from $87 \%$ to $100 \%$ in all 8 domains (data indicators are most desirable when greater than $90 \%$ ). On baseline assessment, the UCG showed slight response inconsistencies with data indicators at $86 \%, 87 \%$, and $88 \%$ in 3 areas. The data indicators for the end-of-study questionnaires were in the $90 \%$ or better category in all but one area, which was $89 \%$. The SF-36v2@ scoring algorithms require a response for at least half of the items in each of the domains for accurate interpretation (37).

As shown in Table 4, at study entry the IG had significantly poorer physical functioning $(\mathrm{p}=0.02)$ as compared to the UCG. The remaining seven of eight domains were comparable between the two groups. The scores of both the IG and the UCG improved over the study period 
in all domains except the General Health domain. For the IG, the post-intervention scores were significantly improved in six domains, while for the UCG, the post-intervention scores were significantly improved in only four domains. For the two composite scores the PCS and the MCS both significantly improved in the IG, while only the MCS significantly improved in the $\mathrm{UCG}($ all $\mathrm{p}=0.05)$.

As shown in Table 5, the IG was below the $50^{\text {th }}$ percentile of the U.S. normative data in six of the eight pre-intervention domains, while the UCG was below the $50^{\text {th }}$ percentile in five of the eight pre-intervention domains. At the conclusion of the study the scores for both groups improved in all domains except the General Health domain. The IG remained below the $50^{\text {th }}$ percentile norm in two areas (physical functioning and physical role) and the UCG remained below the $50^{\text {th }}$ percentile norm in one area (physical role). Overall, we observed a larger percentage improvement, compared to U.S. norms in the IG, as compared to the UCG.

\section{Depression (Table 5):}

At the beginning of this study, $61 \%$ of the UCG answered "yes" when asked if they were experiencing depression, as compared to $45 \%$ of the IG. At the end-of-the study, the SF-36v2® scoring identified $15 \%$ of the IG and $9 \%$ of the UCG as at-risk for depression as compared to the general U.S. population. In both the IG and the UCG, those with higher mental health composite scores reported less depression, higher physical functioning scores and had lower stage of disease at the end of the study.

\section{Survival (Figures 4a and 4b):}

Overall survival during the study period for all patients with resected PPC did not differ between the IG (range: 72 days -2.3 years) and the UCG (range: 44 days -2.3 years). As of this report median survival has not been reached in either group (See Figure 4a). Using Cox regression to compare survival only in cases of resected pancreatic adenocarcinoma, we found a 
hazard ratio of 1.3 (CI $0.7-2.5$ ) for the UCG as compared to the IG, indicating a small trend towards a survival advantage in the IG (see Figure $4 \mathrm{~b} ; \mathrm{p}=0.56$ ).

\section{Symptom Analysis (Table 6):}

We examined the cohort's symptom profile to determine which symptoms occurred at the same point in time. Cluster analysis after the 3 month time point was used to identify the presence of symptom clusters. Overall the cohort reported an average of 4.5 out of ten symptoms, with $92 \%$ reporting 2 or more symptoms at a mean of 3 months after resection. The five most commonly reported symptoms were fatigue, pain, depression, anxiety and weakness. The UCG reported a greater frequency of symptoms compared to the IG patients, but not significantly more. Using hierarchical cluster analysis, three mutually exclusive symptom groupings were identified in the cohort. The first and most prevalent symptom cluster grouping $(n=61)$ included fatigue (97\%), bodily pain (72\%), depression $(69 \%)$, weakness $(67 \%)$, and anxiety (64\%), as well as trouble sleeping (30\%) and shortness of breath (20\%). Patients in this first cluster have not yet reached median survival (greater than 22 months). Given the high prevalence of fatigue in this first cluster we then re-analyzed this seven-symptom cluster without the symptom of fatigue and no sub-grouping was present, suggesting that fatigue is the central component of this PPC patient cluster, and that these symptoms may share a common biological mechanism. The second symptom cluster grouping $(n=33)$, represented a group of sicker patients and the cluster contained only fatigue (18\%) and shortness of breath (6\%) as the predominant symptoms. Despite their low symptom reporting, patients in this second grouping experienced poorer survival (median survival $=9.3$ months) had a more advanced stage of cancer, had poorer baseline and final ECOG scores, and fewer received adjuvant therapy. The third and least prevalent symptom cluster grouping $(\mathrm{n}=8)$ included fatigue $(88 \%)$, diarrhea $(88 \%)$, pain $(63 \%)$ anxiety $(38 \%)$ and trouble sleeping (13\%). This third group also had poorer survival than the first group, with a median survival of only 10.7 months. 


\section{DISCUSSION}

This is the first study to date to prospectively evaluate the effects of a structured homewalking program in patients following resection for pancreatic and periampullary adenocarcinoma. Patients were approached after recovery from major pancreatic resection, signed appropriate informed consent, completed various validated assessment tools, and were randomly allocated to the intervention group (IG) or the usual care group (UCG). At the conclusion of the study the IG and the UCG appeared to be well matched with regard to demographics, pathological parameters and baseline clinical characteristics (Table 1). Of note, the IG at the end of the study reported walking over twice as far as the UCG (Table 2; 2 miles for the IG vs. 1 mile for the UCG) and were significantly more likely to still be walking at the study end $(80 \%$ versus $58 \%$ in the UCG; $\mathrm{p}=0.04)$. Using the various validated assessment tools (FVAS, FFS, PVAS and SF-36) the IG was observed to have improvements in fatigue, pain, and physical composite scores that exceeded the improvements observed in the UCG. Of note, the IG received an exercise prescription, a detailed exercise booklet and a monthly phone call. The phone call was a key component of the protocol. Not only did the phone call serve as a reminder to walk or exercise, but it was also an opportunity to learn about their life after PPC resection. The phone call provided us an entry into the details and personal response to their adjuvant therapy. The monthly phone call to encourage the IG to walk and exercise, along with the monthly log sheets, we believe were critical to the success of the program. Patients in general were very grateful for the follow-up phone call and it provided us with a great deal of information on how they were faring in their recovery. The structured walking program (Figure 3), reinforced with regularly scheduled telephone follow-up by a knowledgeable advanced practice nurse, likely improved adherence with the protocol. Even for IG patents that did not walk or exercise, the phone call was quite important in establishing rapport and getting a glimpse of surviving life following resection of PPC. One practice implication of the current study is that 
this type of telephone follow-up should be implemented when feasible, as it appeared to be much appreciated by the patients and served as a tangible reminder to persist with their exercise prescription.

We found that the adverse symptoms related to chemotherapy (such as nausea, vomiting, and anorexia) peaked one to two days after chemotherapy and dissipated in 1 to 3 days, with a return to baseline symptom status thereafter. Patients in the IG typically had an "exercise break" during this period and many returned to walking the following week. We noted that symptoms reported during adjuvant therapy often reflect an increased symptom intensity due to the treatment. Therefore it is important to time the collection of data to minimize capture at these peak times (unless the intent of the study is to capture therapy-related side effects).

Since clinical practice is unlikely to be altered by this one study and replication studies are needed to truly affect practice, a prospective randomized longitudinal study which includes a walking component and an objective measure of energy expenditure (such as the use of a pedometer, "body bug" or other electronic monitoring device to quantify movement) is needed. Support for recommending exercise in cancer survivors is abundant. Schmitz et al conducted a systematic review and meta-analysis including data extracted from 22 high-quality studies (38). They found qualitative and quantitative evidence of a small to modest effect of physical activity on cardiopulmonary fitness during and following cancer treatment, with improvement in physiologic outcomes and symptoms. They concluded that physical activity is well-tolerated by cancer patients during cancer treatment and afterwards.

We found only mild to moderate pain in the patients enrolled in this study. This may have been due to the exclusion of patients with stage 4 disease, routine prescribing of oral pain meds, failure to recruit patients with serious perioperative complications, and the inclusion of a number of patients who had received a celiac plexus alcohol block at the time of resection (as part of an ongoing double blinded RCT at our institution). One future approach would be to 
exclude those on pain medications or query the patients more often and keep a diary of pain medications taken. We were concerned about "response burden" in this population, and therefore we kept the number of measurement tools to a minimum.

While this RCT offers evidence of the benefit of a home-walking program in resected PPC patients and preliminary evidence of the existence of a fatigue-related symptom cluster (fatigue, bodily pain, depression, weakness and anxiety), there are several limitations that must be acknowledged. First, the sample was predominantly Caucasian which limits applicability to other racial and socioeconomic groups. A second limitation relates to the patients' pre-diagnosis physical activity and our lack of stratification for this variable. It could be suggested that people who were more physically active before their PPC diagnosis may have been more physically active after their diagnosis and more willing to participate in the study. While this is a possibility, prior exercise experience was not an inclusion criterion of the study, and group designation was randomly assigned. Such randomization should have evenly distributed the pre-diagnosis exercisers between the IG and the UCG. Of note Meyerhardt et al in their investigation of colorectal cancer survivors using data from the Nurses Health Survey, found that pre-diagnosis exercise did not affect colo-rectal specific mortality or overall mortality, but post-diagnosis exercise did confer reduced colo-rectal mortality (39). A third limitation (or criticism) of this study is that the monthly phone call received by the IG may have been largely responsible for the observed improvement in symptomatology, as opposed to the walking program. This issue would be well-addressed in a replication study with an "attention control group" where the UCG also receives phone contact, or in a larger prospective longitudinal cohort study utilizing a specific MET (metabolic equivalent task) measurement device and more comprehensive symptom profile tools. A fourth limitation addresses the symptom cluster analysis. In this cohort, symptoms may have been caused by the disease, by the treatment (surgery or adjuvant therapy), or by the various co-morbid conditions such as arthritides, deconditioning or 
cardiovascular disease. As the most predominant fatigue-related cluster (fatigue, bodily pain, depression, weakness and anxiety) was identified cross-sectionally at a mean of 3 months following surgical resection, it is currently not known if this symptom cluster is present at other time points during the resected PPC disease trajectory. Future research studies should be directed to determining the stability of this symptom cluster over time, through longitudinal patient investigations.

In conclusion, this is the first RCT to prospectively evaluate the effects of a structured home-walking program in resected PPC patients and to report that participation confers a significant benefit with regard to fatigue and pain levels, physical functioning and mental health. Although the benefits of exercise programs for cancer patients have been recognized since 1986, the optimal regimen is still unclear $(17,40,41)$. There seems to be little harm and much potential benefit to patients by recommending that resected PPC patients engage in low-tomoderate intensity exercise. Published exercise guidelines for cancer patients emphasize the importance of performing an initial clinical assessment and recommend low-to moderate exercise intensity (16). As more patients with pancreas and periampullary cancers experience improved survival, it is important to assess the impact that survival has on their lives and to strive to return them to their pre-operative performance status. One tangible result of this study is that we now recommend a structured walking prescription to our patients, similar to Figure 3, as part of our patients' discharge instructions. Physicians, nurses, advanced practice nurses, social workers and physical therapists are all instrumental in this process. 


\section{REFERENCES:}

1. American Cancer Society: Cancer facts and figures. American Cancer Society 2011; Atlanta, GA.

2. Cress RD, Yin D, Clarke L, et al. Survival among patients with adenocarcinoma of the pancreas: A population-based study (United States). Ann Surg 2006;17:403-409.

3. Cleary S, Gryfe R, Guindi, M, et al. Prognostic Factors in resected pancreatic adenocarcinoma: analysis of actual 5-yeasr survivors. Am Coll Surg 2004; 198:722-731.

4. Ishikawa O, Ohihashi H, Yamada T, et al. Radical resection for pancreatic cancer. Acta Gastroenterology Belgium 2002; 65(3): 166-170.

5. Sohn TA, Yeo CJ, Cameron JL, et al. Resected adenocarcinoma of the pancreas-616 patients: results, outcomes, and prognostic indicators. J Gastrointest Surg 2000; 4(6):56779.

6. Winter JM, Cameron JL, Campbell KA, et al. 1423 pancreaticoduodenectomies for pancreatic cancer: a single-institution experience. J Gastrointest Surg 2006; 10:11991211.

7. Birkmeyer J, Siewers A, Finlayson E, et al. Hospital volume and surgical mortality in the United States. New England Journal of Medicine 2002; 346(15): 1128-1137.

8. Conlon K, Klemstra D, Brennan M. Long-term survival after curative resection for pancreatic ductal adenocarcinoma: Clinocopathologic analysis of 5-year survivors. Ann Surg 1996; 223(3): 273-279.

9. Luthy C, Cedrasdi C, Pugliesi A, et al. Patients' views about causes and preferences for the management of cancer-related fatigue- a case for non-congruence with physicians. Support Care Cancer 2011;19:363-370.

10. Hoffman M, Ryan JL, Figueroa-Moseley CD, et al. Cancer-related fatigue: the scale of the problem. Oncologist 2007;(Suppl):5-10. 
11. Coleman J, Olsen S, Sauter P et al. The effect of a frequently asked questions module on a pancreatic cancer web site patient/family chat room. Cancer Nursing 2005; 28(6): 460464.

12. Sun V, Ferrell B, Juarez G, et al. Symptom concerns and quality of life in hepatobiliary cancers. Oncology Nursing Forum 2008; 35(3): E45-E52.

13. Muller-Nordhorn J, Roll S, Bohmig M, et al. Health-related quality of life in patients with pancreatic cancer. Digestion 2006; 74(2):118-125.

14. Carlson LE, Angen M, Cullum J, et al. High levels of untreated distress and fatigue in cancer patients. British Journal of Cancer 2004; 90: 2297-2304.

15. Reyes-Gibby CC, Chan W, Abbruzzese JL, et al. Patterns of self-reported symptoms in pancreatic cancer. Journal of Pain and Symptom Management 2007; 34(3):244-252.

16. National Comprehensive Cancer Network (NCCN) Clinical Practice Guidelines in Oncology. Cancer-related fatigue. Version 1.2011. Available at: http://www.nccn.org/professionals/physician_gls/pdf/fatigue.pdf

17. Winningham ML. Strategies for managing cancer-related fatigue syndrome. Cancer 2001; 92(4):988-997.

18. Dimeo F, Rumberger B, Keul J. Aerobic exercise as therapy for cancer fatigue. Med Sci Sports Exerc 1988;30:475-8.

19. Mock V, Pickett M, Ropka M, et al. Fatigue and quality of life outcomes of exercise during cancer treatment. Cancer Practice 2001 ; 9(3):119-127.

20. MacVicar M, Winningham M, Nickel J. Effects of aerobic interval training on cancer patients' functional capacity. Nursing Research 1989; 38: 348-351.

21. Wanchai A, Armer J, Stewart B. Nonpharmacologic supportive strategies to promote quality of life in patients experiencing cancer-related fatigue: A systematic review. CJON 2011; 15(2):203-214. 
22. Dodd MJ, Miaskowski C, Lee KA. Occurrence: symptom clusters. J Natl Cancer Institute $2004 ; 22: 76-8$.

23. Kim JH, McGuire DB, Tulman L, et al. Symptom clusters: concept analysis and clinical implications for cancer nursing. Cancer Nursing 2005; 28 (4): 270-282.

24. Xiao C. (2010). The state of science in the study of cancer symptom clusters. European Journal of Oncology Nursing 2010; 14(5): 417-34.

25. Huang J, Yeo CJ, Sohn, TA, et al. Quality of life and outcomes after pancreaticoduodenectomy. Ann Surg 2000; 231(6): 890-898.

26. McLeod RS. Quality of life, nutritional status and gastrointestinal hormone profile following the Whipple procedure. Annals of Oncology1999;10(Suppl 4):S281 -S284

27. Shaw CM, O'Hanlon DM, McEntee GP. Long-term quality of life following pancreaticoduodenectomy. Hepato-Gastroenterology 2005; 52(63): 927-932.

28. George J. Myra Estrin Levine. In: Nursing theories, $4^{\text {th }}$ Ed 1995; Norwalk, CT: Appleton \& Lange, pp.195-205.

29. Simon R. Design and conduct of clinical trials. In: Cancer principles \& practice of oncology. (DeVita, Hellman, Rosenberg, Eds.). Philadelphia: J.B. Lippincott, 1982, pp.198-225.

30. Mock V, Cameron L, Tompkins C. et al. Every Step Counts: A walking exercise program for individuals with cancer. Baltimore: Johns Hopkins University, 1997.

31. Torrence G, Feeny D, Furlong, W. Visual Analog scales, do they have a role in the measurement of preferences for health states? Medical Decision Making 2001; 21(4): 329334.

32. Yellen S, Cella D, Webster K, et al. Measuring fatigue and other anemia-related symptoms with the functional assessment of cancer therapy (FACT) measurement system. Journal of Pain and Symptom Management 1997; 13(2):63-74. 
33. Oken M, Creech R, Horton D, et al. Toxicity and response criteria of the Eastern Cooperative Oncology Group. American Journal of Clinical Oncology 1982; 5:649-655

34. Ware J, Sherbourne C. The MOS 36-item short-form health survey (SF-36). Conceptual framework and item selection. Medical Care 1992; 30:473-483.

35. Ware J, Kosinki M, Bjorner J, et al. User's manual for the SF-36v2® Health survey. $\left(2^{\text {nd }}\right.$ Ed.). Lncoln, RI: QualityMetric Incorporated, 2007.

36. Roiland RA, Heidrich SM. Symptom clusters and quality of life in older adult breast cancer survivors. Onc Nursing For 2011;38(6):672-679.

37. Kosinski M, Bayliss M, Bjorner J, et al. Improving estimates of SF-36 ${ }^{1}{ }^{\text {health }}$ survey scores for respondents with missing data. Monitor Fall 2000; 8-10.

38. Schmitz KH, Holtzman J, Courneya KS, et al. Controlled physical activity trials in cancer survivors: a systematic review and meta-analysis. Cancer Epi, Biomarkers, Prevention 2005;14(7):1588-1595

39. Meyerhardt JA, Heseltine D, Niedzwiecki D, et al. Impact of physical impact on colon recurrence and survival in patients with stage III colon cancer: findings from CALGB 89803, J Clin Oncol 2006; 24(22):3535-3541.

40. Hanna LR, Avial PF, Meterr JD et al. The effects of a comprehensive exercise program on physical function, fatigue, and mood in patients with various types of cancer. Onc Nursing For 2008;35(3):461-469.

41. Mitchell, S., Beck, S., Hood, L., Moore, K, \& Tanner, E. Putting evidence into practice: Evidence-based interventions for fatigue during and following cancer and its treatment. Clinical Journal of Oncology Nursing, 2007; 11(1): 99-113. 
Table 1: Demographic, Pathologic and Clinical Characteristics of the Intervention Group and the Usual Care Group

\begin{tabular}{|c|c|c|c|}
\hline $\begin{array}{c}\text { Characteristic of Subjects } \\
(\mathrm{N}=102)\end{array}$ & $\begin{array}{l}\text { Intervention Group } \\
(\mathrm{n}=54)\end{array}$ & $\begin{array}{l}\text { Usual Care Group } \\
(\mathrm{n}=\mathbf{4 8})\end{array}$ & $\begin{array}{c}p- \\
\text { value }\end{array}$ \\
\hline Age at Time of Surgery (yrs, range) & $66(38-87)$ & $67(48-91)$ & 0.7 \\
\hline Male $(n, \%)$ & $29(51 \%)$ & $28(49 \%)$ & 0.6 \\
\hline $\begin{array}{l}\text { Race (n): } \\
\text { White } \\
\text { Black, Asian, or Hispanic }\end{array}$ & $\begin{array}{c}52 \\
2\end{array}$ & $\begin{array}{c}45 \\
3\end{array}$ & 0.5 \\
\hline $\begin{array}{l}\text { Diagnosis (n,\%): } \\
\text { Pancreas Ductal Adenocarcinoma } \\
\text { Bile Duct Cancer } \\
\text { IPMN with Cancer } \\
\text { Duodenal Cancer }\end{array}$ & $\begin{array}{l}50(93 \%) \\
1 \\
2 \\
1\end{array}$ & $\begin{array}{r}43(90 \%) \\
2 \\
2 \\
1\end{array}$ & 0.6 \\
\hline $\begin{array}{l}\text { Stage of Cancer }(\mathbf{n}, \%) \text { : } \\
1 \mathrm{a}, \mathrm{b} \\
2 \mathrm{a}, \mathrm{b} \\
3\end{array}$ & $\begin{array}{c}6 \\
44(73 \%) \\
4 \\
\end{array}$ & $\begin{array}{c}6 \\
35(72 \%) \\
7 \\
\end{array}$ & 0.5 \\
\hline $\begin{array}{l}\text { Type of Surgery (n): } \\
\text { Pylorus Preserving Pancreaticoduodenectomy } \\
\text { Classic Whipple } \\
\text { Distal Pancreatectomy }\end{array}$ & $\begin{array}{l}50(93 \%) \\
1 \\
3\end{array}$ & $\begin{array}{c}44(92 \%) \\
1 \\
3\end{array}$ & 0.7 \\
\hline $\begin{array}{l}\text { Co-morbidities (n, \%): } \\
\text { Previous cancers } \\
\text { Diabetes } \\
\text { Hypertension } \\
\text { Coronary Artery Disease } \\
\text { Cigarette smoking } \\
\end{array}$ & $\begin{array}{l}10(19 \%) \\
17(31 \%) \\
35(65 \%) \\
14(23 \%) \\
30(56 \%) \\
\end{array}$ & $\begin{array}{l}6(11 \%) \\
15(32 \%) \\
25(52 \%) \\
11(26 \%) \\
30(63 \%) \\
\end{array}$ & $\begin{array}{l}0.4 \\
0.9 \\
0.2 \\
0.7 \\
0.5\end{array}$ \\
\hline \multicolumn{4}{|l|}{ Laboratory Values Pre-op (mean): } \\
\hline Albumin (mg/dl) & 3.6 & 3.4 & 0.3 \\
\hline Glucose (mg/dl) & 142 & 139 & 0.8 \\
\hline Total bilirubin (mg/dl) & 3.4 & 2.9 & 0.6 \\
\hline Hemoglobin $(\mathrm{g} / \mathrm{dl})$ & 12 & 12 & 0.4 \\
\hline CEA (mcg/L, range) & $3.4(0.5-31)$ & $3.6(0-17.5)$ & 0.8 \\
\hline CA 19-9 $(\mathrm{U} / \mathrm{ml}$, range $)$ & $950(1-19,867)$ & $2000(0.8-37,333)$ & 0.6 \\
\hline BMI baseline (mean, range) & $28(17-49)^{*}$ & $26(19-42)$ & 0.02 \\
\hline $\begin{array}{c}\text { Adjuvant Therapy (N,\%) (CT, RT or both) } \\
\text { (None) }\end{array}$ & $\begin{array}{l}39(72 \%) \\
15(28 \%)\end{array}$ & $\begin{array}{l}35(73 \%) \\
13(27 \%)\end{array}$ & 0.5 \\
\hline $\begin{array}{l}\text { Most Common Chemotherapy (n,\%) } \\
\text { Gem +/- } 5 \text { FU (missing data } 3 \text { subjects, } 2 \text { had } \\
\text { other CT) }\end{array}$ & $34 / 53=64 \%$ & $24 / 46=52 \%$ & 0.2 \\
\hline Deaths: in study period (n, \%) & $7(13 \%)$ & $8(17 \%)$ & 0.6 \\
\hline Deaths: overall $(\mathrm{n}, \%)$ & $12(22 \%)$ & $15(31 \%)$ & 0.6 \\
\hline
\end{tabular}

$* \mathrm{p}=0.02$ compared to the UCG 
Table 2: Comparison of Walking Program Characteristics: Intervention Group Compared to Usual Care Group

\begin{tabular}{|c|c|c|c|}
\hline Walking Program & Intervention Group & Usual Care Group & $p$-value \\
\hline $\begin{array}{l}\text { IG and UCG Baseline Walk } \\
(\text { feet, range })(I G=54, U C G=48)\end{array}$ & $\begin{array}{c}913(10-5280) \\
0.17 \text { miles } \\
\end{array}$ & $\begin{array}{c}, 404(10-7920) \\
0.27 \text { miles } \\
\end{array}$ & 0.1 \\
\hline $\begin{array}{l}\text { IG First Month Walk } \\
\text { (feet, range) }(\mathrm{n}=38)\end{array}$ & $\begin{array}{c}13,412(420-150,797) \\
2.5 \text { miles } \\
\end{array}$ & --- & --- \\
\hline $\begin{array}{l}\text { IG Second Month Walk } \\
\text { (feet, range) }(\mathrm{n}=29)\end{array}$ & $\begin{array}{c}5,914(330-73,900) \\
1.1 \text { miles }\end{array}$ & --- & --- \\
\hline $\begin{array}{l}\text { IG and UCG Final Walk } \\
\text { (feet, range) }(\mathrm{IG}=31, \mathrm{UCG}=18)\end{array}$ & $\begin{array}{l}10,772(420-147,840) \\
2 \text { miles }\end{array}$ & $\begin{array}{c}5,219(1,320-63,360) \\
1 \text { mile } \\
\end{array}$ & 0.1 \\
\hline Follow-up time (weeks, mean) & 12 & 13 & 0.9 \\
\hline $\begin{array}{l}\text { Subjects that Completed Study } \\
\text { ( } \mathrm{n}=\text { eligible to complete study, i.e. alive, \%) }\end{array}$ & $41 / 47(87 \%)$ & $35 / 40(88 \%)$ & 0.7 \\
\hline $\begin{array}{l}\text { Subjects Still Walking at End-of-Study } \\
(\mathrm{n}, \%)\end{array}$ & $33 / 41=80 \% *$ & $18 / 31=58 \%$ & 0.04 \\
\hline
\end{tabular}

$*$ p-value $=0.04$ compared to the UCG 
Table 3: Fatigue, Pain, and ECOG Performance Status: Intervention Group Compared to Usual Care Group

\begin{tabular}{|c|c|c|c|c|}
\hline Outcome Measures & \multicolumn{2}{|c|}{$\begin{array}{l}\text { Intervention Group } \\
(n=54) \quad(n=47)\end{array}$} & \multicolumn{2}{|c|}{$\begin{array}{l}\text { Usual Care Group } \\
(\mathrm{n}=\mathbf{4 8}) \quad(\mathrm{n}=\mathbf{4 0})\end{array}$} \\
\hline $\begin{array}{l}\text { Fatigue Visual Analog Scale (FVAS) } \\
\text { (mean, range 0-10) } \\
\text { (Higher Score }=\text { More Fatigue) }\end{array}$ & $\frac{\text { Pre }}{4.8}$ & $\frac{\text { Post }}{3.5^{*}}$ & $\frac{\text { Pre }}{4.7}$ & $\frac{\text { Post }}{3.8}$ \\
\hline $\begin{array}{l}\text { FACIT- Fatigue Scores (FFS) } \\
\text { (mean, range 0-52) } \\
\text { (Higher Score = Less Fatigue) }\end{array}$ & 27 & $36^{*}$ & 30 & 34 \\
\hline $\begin{array}{l}\text { Pain Visual Analog Scores (PVAS) } \\
\text { (mean, range } 0-10) \\
\text { (Higher Score }=\text { More Pain) }\end{array}$ & 2.9 & $1.6^{*}$ & 2.9 & $1.8^{\star}$ \\
\hline $\begin{array}{l}\text { ECOG Stage, }(\text { mean, range) } \\
\text { (Score } 0=\text { no limitations }-5=\text { dead) }\end{array}$ & $\begin{array}{c}1.6 \\
(1-3)\end{array}$ & $\begin{array}{c}1.5 \\
(0-5)\end{array}$ & $\begin{array}{c}1.5 \\
(0-3)\end{array}$ & $\begin{array}{l}1.8 \\
(0-5)\end{array}$ \\
\hline
\end{tabular}

${ }^{*} \mathrm{p}<=0.05$, paired t-tests used in analysis

$E C O G=$ Eastern Cooperative Oncology Group 
Table 4: Short Form-36 Health Survey® Pre / Post Intervention Scores

\begin{tabular}{|l||c|c|c|c|}
\hline SF-36 Health Survey® & \multicolumn{2}{|c|}{ Intervention Group } & \multicolumn{2}{c|}{ Usual Care Group } \\
\hline 8 Domains & $\begin{array}{c}\text { Pre } \\
(\mathbf{n = 5 4})\end{array}$ & $\begin{array}{c}\text { Post } \\
(\mathbf{n}=39)\end{array}$ & $\begin{array}{c}\text { Pre } \\
(\mathbf{n}=48)\end{array}$ & $\begin{array}{c}\text { Post } \\
(\mathbf{n}=35)\end{array}$ \\
\hline Physical Functioning & $34^{*}$ & $43^{* *}$ & 39 & $43^{* *}$ \\
\hline Role-Physical & 34 & $41^{* *}$ & 38 & 40 \\
\hline Bodily Pain & 47 & $53^{* *}$ & 42 & $50^{* *}$ \\
\hline General Health & 48 & 46 & 48 & 44 \\
\hline Vitality & 44 & $50^{* *}$ & 43 & $46^{* *}$ \\
\hline Social Functioning & 37 & $46^{* *}$ & 39 & $45^{* *}$ \\
\hline Role-Emotional & 42 & 47 & 43 & 47 \\
\hline Mental Health & 45 & $51^{* *}$ & 44 & 48 \\
\hline SF-36v2 ${ }^{*}$ Health Survey Scores $\dagger * *$ & & & & \\
\hline Physical Composite Scores (PCS) \\
Mental Composite Scores (MCS)
\end{tabular}

*Pre scores: IG to UCG, $\mathrm{p}<=0.05$,

$* *$ Paired pre/post t-tests, $\mathrm{p}<=0.05$.

SF-36v2@ Scoring 20-80: higher scores = better functioning 
Table 5: Percent of Study Participants below General Population Norms* on Eight Domains at Baseline and at End of Study

\begin{tabular}{|c|c|c|c|c|c|c|}
\hline $\begin{array}{l}\text { SF-36 Health } \\
\text { Surveyv2® }\end{array}$ & \multicolumn{3}{|c|}{$\begin{array}{c}\text { Intervention Group } \\
(\% \text { below Norm })\end{array}$} & \multicolumn{3}{|c|}{$\begin{array}{l}\text { Usual Care Group } \\
\text { (\% below Norm) }\end{array}$} \\
\hline Domains & $\begin{array}{c}\text { Pre } \\
(n=54)\end{array}$ & $\begin{array}{c}\text { Post } \\
(n=39)\end{array}$ & $\begin{array}{l}\text { Improved / } \\
\text { Worsened }\end{array}$ & $\begin{array}{c}\text { Pre } \\
(n=49)\end{array}$ & $\begin{array}{c}\text { Post } \\
(n=35)\end{array}$ & $\begin{array}{l}\text { Improved/ } \\
\text { Worsened }\end{array}$ \\
\hline Physical Functioning & $78 \%$ & $51 \%$ & $+27 \%$ & $78 \%$ & $49 \%$ & $+\mathbf{2 9} \%$ \\
\hline Role-Physical & $83 \%$ & $62 \%$ & $+21 \%$ & $65 \%$ & $63 \%$ & $+2 \%$ \\
\hline Bodily Pain & $50 \%$ & $23 \%$ & $+27 \%$ & $59 \%$ & $31 \%$ & $+28 \%$ \\
\hline General Health & $35 \%$ & $44 \%$ & $-9 \%$ & $31 \%$ & $43 \%$ & $-12 \%$ \\
\hline Vitality & $57 \%$ & $31 \%$ & $+26 \%$ & $39 \%$ & $37 \%$ & $+2 \%$ \\
\hline Social Functioning & $65 \%$ & $33 \%$ & $+32 \%$ & $59 \%$ & $40 \%$ & $+19 \%$ \\
\hline Role-Emotional & $57 \%$ & $33 \%$ & $+24 \%$ & $49 \%$ & $46 \%$ & $+3 \%$ \\
\hline Mental Health & $44 \%$ & $28 \%$ & $+16 \%$ & $51 \%$ & $31 \%$ & $+20 \%$ \\
\hline $\begin{array}{l}\text { Physical Function } \\
\text { Composite Score }\end{array}$ & $70 \%$ & $46 \%$ & $+24 \%$ & $69 \%$ & $54 \%$ & $+15 \%$ \\
\hline $\begin{array}{l}\text { Mental Health } \\
\text { Composite Score }\end{array}$ & $56 \%$ & $31 \%$ & $+25 \%$ & $47 \%$ & $26 \%$ & $+21 \%$ \\
\hline Depression, At-Risk & $24 \%$ & $15 \%$ & $+9 \%$ & $18 \%$ & $9 \%$ & $+9 \%$ \\
\hline
\end{tabular}

* Norms from the 1998 U.S. National Survey of Functional Health Status (Reference \#35). 
Table 6: End-of-Study Symptom Profile in Intervention Group versus Usual Care Group

\begin{tabular}{|l|c|c|c|}
\hline \multicolumn{1}{|c|}{$\begin{array}{c}\text { Symptoms } \\
(\mathbf{n , \%})\end{array}$} & $\begin{array}{c}\text { Intervention } \\
\text { Group }\end{array}$ & $\begin{array}{c}\text { Usual Care } \\
\text { Group }\end{array}$ & $\boldsymbol{p}$-value \\
\hline Fatigue & $37 / 43(86 \%)$ & $34 / 37(92 \%)$ & $\mathbf{0 . 2}$ \\
\hline Bodily Pain & $18 / 43(42 \%)$ & $21 / 35(60 \%)$ & $\mathbf{0 . 2}$ \\
\hline Weakness & $25 / 43(58 \%)$ & $11 / 37(30 \%)$ & $\mathbf{0 . 9}$ \\
\hline Depression & $19 / 43(44 \%)$ & $22 / 36(61 \%)$ & $\mathbf{0 . 3}$ \\
\hline Anxiety & $16 / 43(37 \%)$ & $26 / 37(70 \%)$ & $\mathbf{0 . 6}$ \\
\hline Diarrhea & $12 / 43(30 \%)$ & $11 / 36(31 \%)$ & $\mathbf{0 . 9}$ \\
\hline Constipation & $6 / 43(14 \%)$ & $7 / 37(19 \%)$ & $\mathbf{0 . 6}$ \\
\hline Trouble Sleeping & $12 / 40(30 \%)$ & $10 / 35(29 \%)$ & $\mathbf{0 . 9}$ \\
\hline Weight Loss & $7 / 43(16 \%)$ & $7 / 37(19 \%)$ & $\mathbf{0 . 6}$ \\
\hline Shortness of Breath & $9 / 43(21 \%)$ & $5 / 32(14 \%)$ & $\mathbf{0 . 4}$ \\
\hline
\end{tabular}




\section{FIGURE LEGENDS}

Figure 1. Flow diagram of study participants. Of the 165 potentially eligible patients, 118 were approached for study inclusion, 110 were enrolled, and data were sufficient for analysis in 102 patients.

Figure 2. Basic design of the study. Following randomization the patients were allocated to either the Intervention Group or the Usual Care Group. $(\mathrm{PPC}=$ pancreas and periampullary cancer; FVAS = Fatigue Visual Analog Scale; PVAS = Pain Visual Analog Scale; FFS = FACIT-Fatigue Scale; SF-36 = Short Form -36 v $2 ®$ questionnaire)

Figure 3. Example of the graduated walking prescription given to patients allocated randomly to the Intervention Group.

Figure 4a. Kaplan-Meier overall survival curves for the Intervention Group $(-, \mathrm{n}=54)$ and the Usual Care Group (---, $\mathrm{n}=48)$ for all patients with resected pancreatic and periampullary adenocarcinoma. Median survival has not been reached in either group, and the curves are not significantly different. Time zero is the date of resection.

Figure 4b. Kaplan-Meier overall survival curves for the Intervention Group $(-, n=50)$ and the Usual Care Group (---, $\mathrm{n}=43)$ for patients with resected pancreatic adenocarcinoma. Median survival has not been reached in either group, and the curves are not significantly different. Time zero is the date of resection. 
Figure 1: Flow Diagram of Study Participants

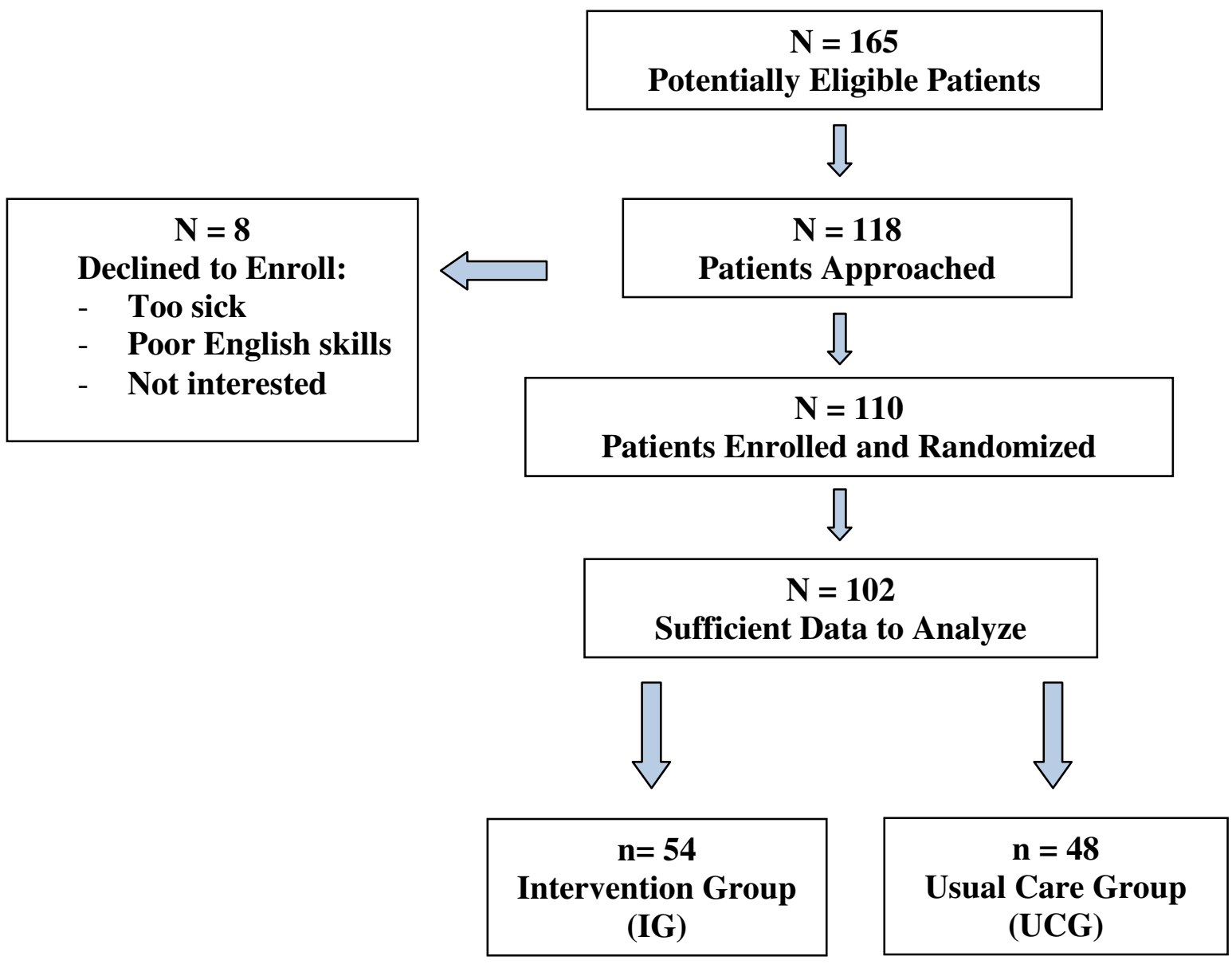


Figure 2: Study Design

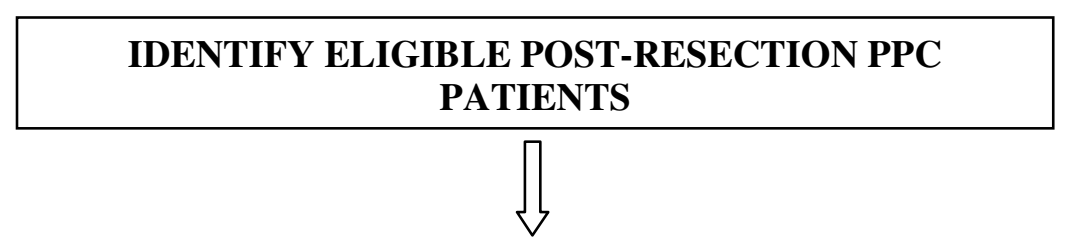

RANDOMIZE TO INTERVENTION GROUP or USUAL CARE GROUP

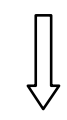

\section{INTERVENTION GROUP:}

- Perform baseline assessment and walk

- FVAS, PVAS,FFS, SF-36

- Exercise booklet and prescription before discharge

- Monthly exercise logs

- Monthly follow-up phone call

- Repeat questionnaires at 3 - 6 months

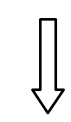

\section{USUAL CARE GROUP:}

-Perform baseline assessment and walk - FVAS, PVAS, FFS, SF-36

- No monthly phone calls

- Perform usual activity/exercise

- Repeat questionnaires at 3 - 6 months 
Figure 3: Walking Prescription

Name:

Your Nurse Practitioner:

Date:

\begin{tabular}{|l|llc|}
\hline Month 1: & Phase 1: Warm-up & Walk slowly for: & 5 minutes \\
& $\begin{array}{l}\text { Phase 2: Brisk Walking } \\
\text { Phase 3: Cool Down }\end{array}$ & Walk more briskly for: & $\mathbf{1 0}$ minutes \\
& Phase 1: Warm-up & Walk slowly for: & 5 minutes \\
& Phase 2: Brisk Walking & Walk more briskly for: & $\mathbf{2 0}$ minutes \\
& Phase 3: Cool Down & Walk slowly for: & 5 minutes \\
\hline Month 2: & Phase 1: Warm-up & Walk slowly for: & 5 minutes \\
& Phase 2: Brisk Walking & Walk more briskly for: & $\mathbf{2 5 - 3 0}$ minutes \\
& Phase 3: Cool Down & Walk slowly for: & 5 minutes \\
\hline
\end{tabular}

How Often to Walk? Start with short distances every day and build-up to $3 \mathbf{- 5}$ times per week. 
Figure 4a: Total Cohort, Survival by Group

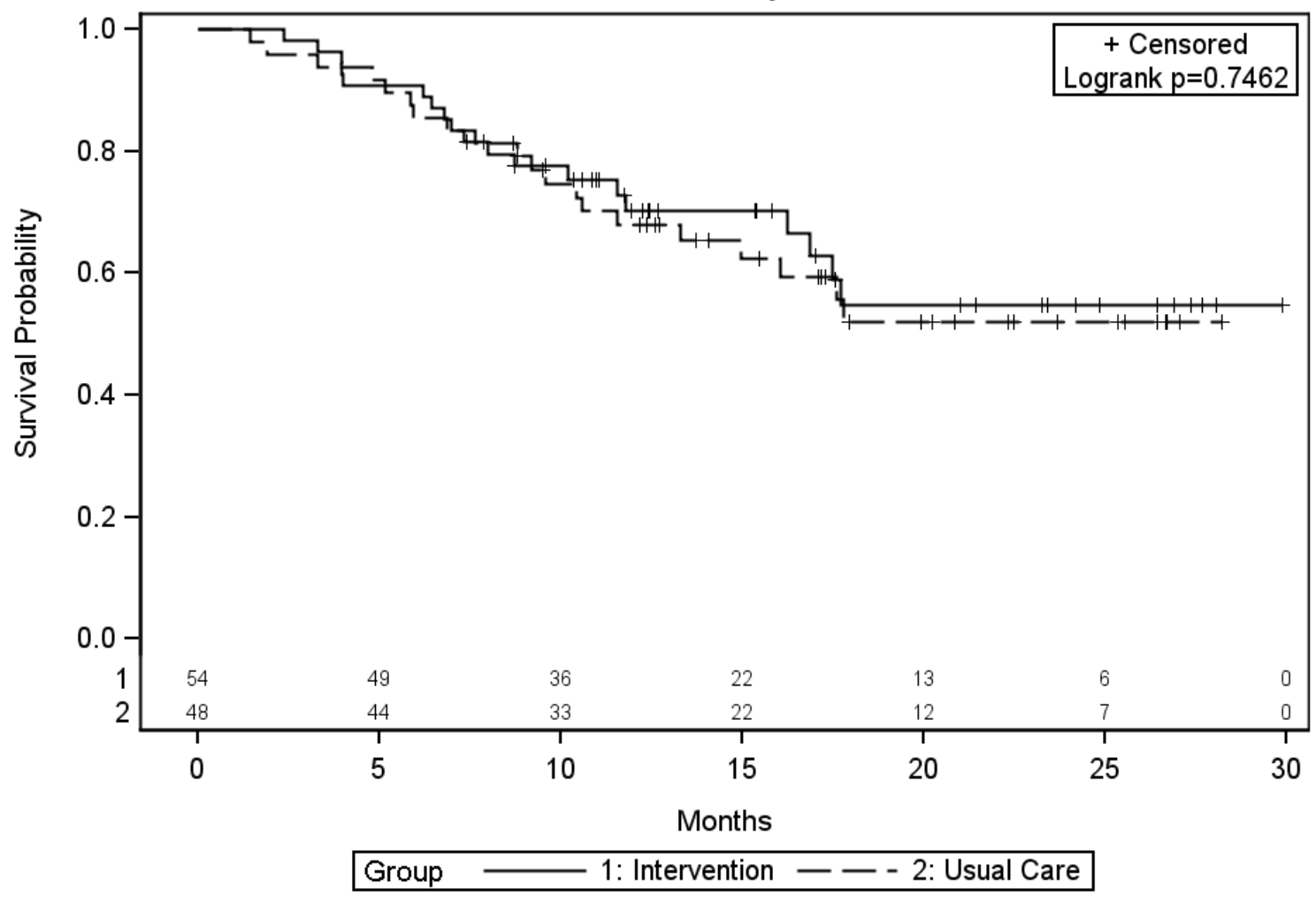


Figure 4b: Resected Pancreas Cancer Patients Only, Survival by Group

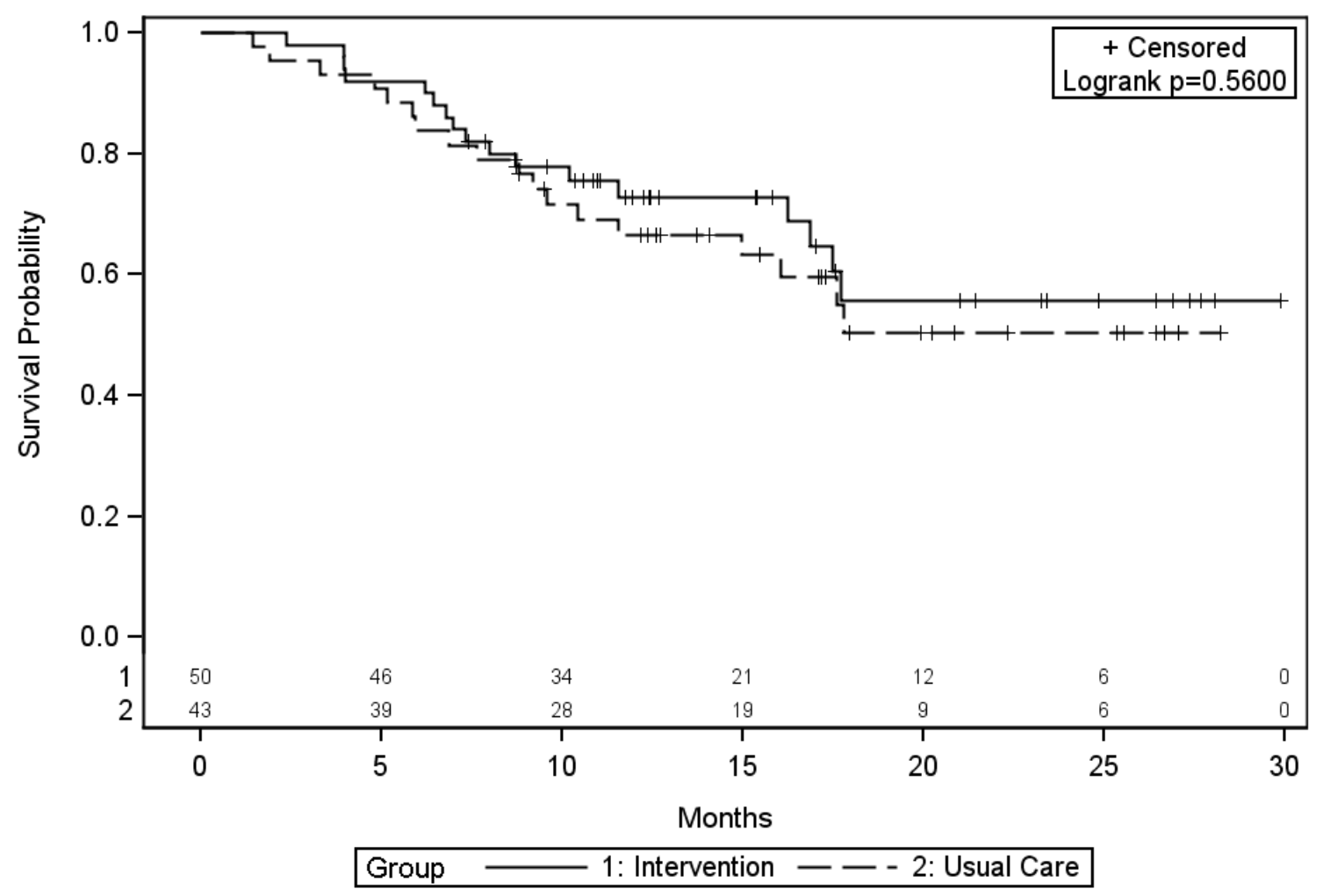

